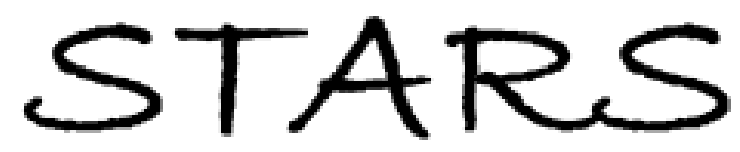

University of Central Florida

STARS

$1-1-2005$

\title{
Growth and characterization of sputtered BSTO/BaM multilayers
}

S. Srinath

N. A. Frey

R. Heindl

H. Srikanth

K. R. Coffey

University of Central Florida

See next page for additional authors

Find similar works at: https://stars.library.ucf.edu/facultybib2000 University of Central Florida Libraries http://library.ucf.edu

This Article; Proceedings Paper is brought to you for free and open access by the Faculty Bibliography at STARS. It has been accepted for inclusion in Faculty Bibliography 2000 s by an authorized administrator of STARS. For more information, please contact STARS@ucf.edu.

\section{Recommended Citation}

Srinath, S.; Frey, N. A.; Heindl, R.; Srikanth, H.; Coffey, K. R.; and Dudney, N. J., "Growth and characterization of sputtered BSTO/BaM multilayers" (2005). Faculty Bibliography 2000s. 5694.

https://stars.library.ucf.edu/facultybib2000/5694

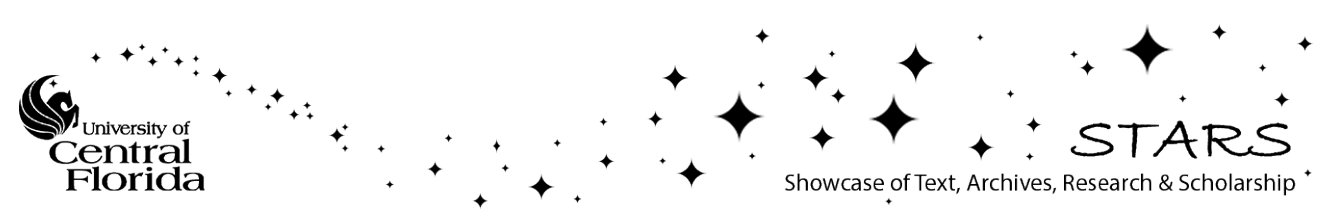




\section{Authors}

S. Srinath, N. A. Frey, R. Heindl, H. Srikanth, K. R. Coffey, and N. J. Dudney 


\section{Growth and characterization of sputtered BSTO/BaM multilayers}

Cite as: J. Appl. Phys. 97, 103115 (2005); https://doi.org/10.1063/1.1853874

Published Online: 11 May 2005

S. Srinath, N. A. Frey, R. Heindl, H. Srikanth, K. R. Coffey, and N. J. Dudney

\section{ARTICLES YOU MAY BE INTERESTED IN}

Multiferroic magnetoelectric composites: Historical perspective, status, and future directions Journal of Applied Physics 103, 031101 (2008); https://doi.org/10.1063/1.2836410

Growth and characterization of BSTO/hexaferrite composite thin films

Journal of Applied Physics 93, 7999 (2003); https://doi.org/10.1063/1.1543129

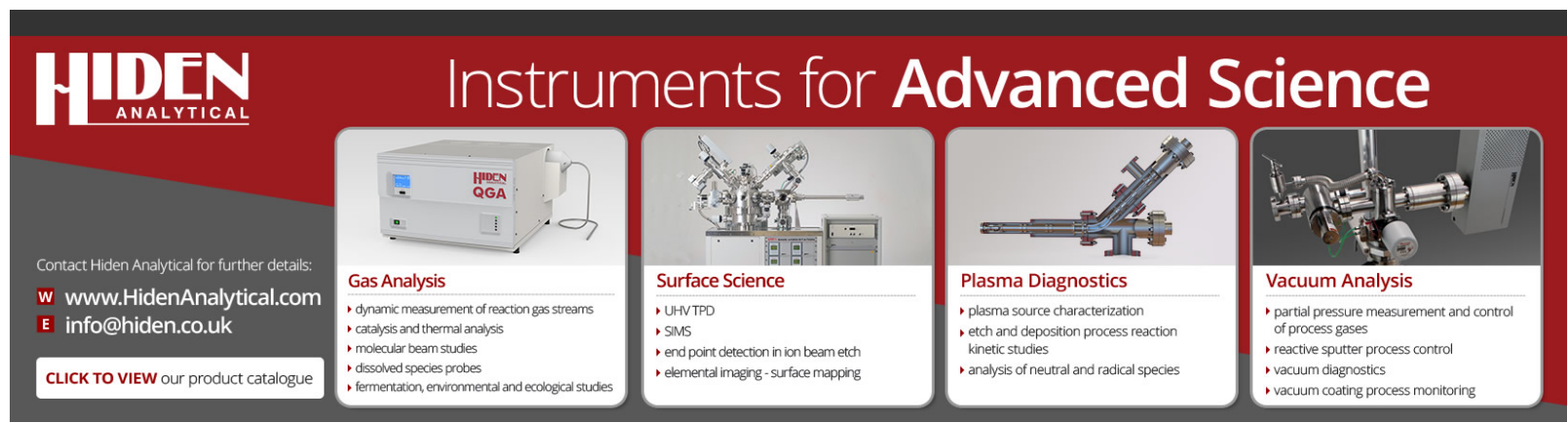




\title{
Growth and characterization of sputtered BSTO/BaM multilayers
}

\author{
S. Srinath, N. A. Frey, R. Heindl, and H. Srikanth ${ }^{\text {a) }}$ \\ Department of Physics, University of South Florida, Tampa, Florida 33620 \\ K. R. Coffey \\ AMPAC, University of Central Florida, Orlando, Florida 33620 \\ N. J. Dudney \\ Condensed Matter Sciences Division, Oak Ridge National Lab, Oak Ridge, Tennessee 37831
}

(Presented on 9 November 2004; published online 11 May 2005)

\begin{abstract}
Multilayers of $\mathrm{Ba}_{0.5} \mathrm{Sr}_{0.5} \mathrm{TiO}_{3}$ (BSTO) and $\mathrm{BaFe}_{12} \mathrm{O}_{19}$ (BaM), with tunable permeability and permittivity are attractive systems for radio frequency and microwave applications. We have grown multilayers of $\mathrm{BSTO}$ and $\mathrm{BaM}$ using magnetron sputtering on $\mathrm{Al}_{2} \mathrm{O}_{3}$ substrates. Film growth conditions such as sputtering parameters were optimized to obtain high quality multilayers. X-ray diffraction established that both BSTO and BaM were formed and cross-sectional SEM studies showed sharp interfaces between BSTO and BaM layers. Magnetization showed a large coercivity ( 2000 Oe) consistent with the hexaferrite component. The hysteresis loops also revealed the distinct influence of magnetocrystalline and shape anisotropies at different temperatures. (C) 2005 American Institute of Physics. [DOI: 10.1063/1.1853874]
\end{abstract}

\section{INTRODUCTION}

Magneto-dielectric composites and multilayered materials have been studied over the years as attractive systems for tunable applications where the individual dielectric constants and permeabilities of the components could be tuned by externally applied electrical and magnetic bias. Recently, renewed interest in systems classified as ferroelectromagnets or multiferroics has opened new avenues to investigate the subtle coupling between the ferroelectric and magnetic components in these materials. ${ }^{1,2}$ The fundamental interest in multiferroics is due to the coexistence of both ferroelectric and ferromagnetic ordering in the same system. This presents a new property of reverse tunability. In other words, the permeability can respond to electrical bias and the dielectric constant tuned by a magnetic field. This discovery has further highlighted the promise of magnetoelectric materials in general for frequency agile, tunable applications in rf and microwave devices.

Most of the multiferroics known at present are ferroelectric-antiferromagnetic systems. This results in a conspicuous absence of response to magnetic fields without spontaneous magnetization. In contrast, ferromagnetic materials mostly are centrosymmetric without any observable spontaneous polarization rendering them ineffective for multiferroics applications. As an alternative, it is prudent to fabricate composite multilayer structures of ferroelectricferromagnetic compounds using different growth techniques with growth parameters optimized based on thickness, microstructure, grain size and shape. While ferrite-ferroelectric materials have been investigated mainly with inverse spinel class of ferrites, ${ }^{3}$ there has been relatively little effort in development of composites in the technologically important

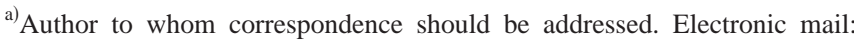
sharihar@cas.usf.edu
}

class of hexagonal ferrites. Hexaferrites exhibit better magnetic response in the $\mathrm{GHz}$ region ${ }^{4}$ compared to spinel ferrites because of their higher anisotropy and larger ferromagnetic resonance (FMR) frequencies and are thus useful in microwave applications. Moreover, the sizable coercivity ( 2000 Oe $)$ in hexaferrites can also be utilized to self-bias nonreciprocal rf devices that require magnetic fields such as phase shifters, circulators, etc. ${ }^{5}$

With the above desirable characteristics in mind, we have chosen to grow multilayers of $\mathrm{BaFe}_{12} \mathrm{O}_{19}(\mathrm{BaM})$ and $\mathrm{BaSr}_{0.5} \mathrm{Ti}_{0.5} \mathrm{O}_{3}$ (BSTO), and optimize the microstructure and magnetic properties. $\mathrm{BaFe}_{12} \mathrm{O}_{19}$ typically has a high permeability $(>100)$ and $\mathrm{BaSr}_{0.5} \mathrm{Ti}_{0.5} \mathrm{O}_{3}$ has a large dielectric constant $(>100)$. These values can be controlled somewhat by the growth conditions like annealing, texturing, grain size, etc. The application potential of both the above materials is well known. BSTO has the potential to be used in thin film capacitors, tunable filters, ${ }^{6}$ and antennas. Even though, individually the properties of BSTO and BaM materials are quite well known, ${ }^{7-13}$ not many reports ${ }^{14-16}$ are available on composite layer growth of these important materials. In this paper we report our successfully optimized growth conditions needed for the growth of quality multilayers of BaM and BSTO. To our knowledge, this is the first such report on BSTO/BaM multilayered films.

In the past we reported on the growth conditions and properties of cosputtered BSTO/BAM films (2 microns thick). ${ }^{14}$ Here the effect of growth and microstructure on the magnetic properties is studied for the multilayers grown on alumina substrates. A key result is that the coupling of the ferroelectric and magnetic layers induces a change in the bulk magnetization as compared to a pure BaM.

\section{EXPERIMENT}

Trilayers of BSTO and BaM are deposited from 3-in. ceramic sputter targets (STMC, Ohio) on polycrystalline Al 


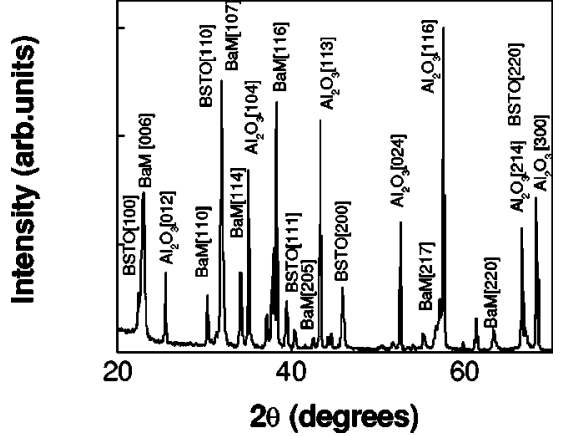

FIG. 1. X-ray diffraction scan of BSTO/BaM multilayer grown on an $\mathrm{Al}_{2} \mathrm{O}_{3}$ substrate at RT and postannealed at $1000^{\circ}$.

substrates using a magnetron sputtering system in the $\mathrm{BaM} /$ $\mathrm{BSTO} / \mathrm{BaM}$ structure. To study the effect of magnetoelastic coupling in multilayers, we have also grown the pure films of BSTO and BaM for comparative analysis under identical deposition conditions. The films were rf sputter deposited at room temperature in an argon pressure of $4 \mathrm{mT}$ with argon gas flow of $20 \mathrm{sccm}$. The rf power used was $70 \mathrm{~W}$. The induced target bias voltage for BaM and BSTO was $302 \pm 3 \mathrm{~V}$ and $196 \pm 2 \mathrm{~V}$, respectively. The as-deposited films (multilayers and also the pure films) were amorphous and were subsequently annealed at $1000{ }^{\circ} \mathrm{C}$ in the presence of 2-3 psi oxygen pressure to get the desired crystalline phases. Films were also deposited in the microstrip structure so as to measure the rf properties. The films were structurally characterized by XRD, SEM, AFM and the magnetic characterization was done using a commercial Physical Property Measurement System (PPMS) from Quantum Design.

\section{RESULTS AND DISCUSSION}

Figure 1 shows the representative XRD pattern for an annealed $\mathrm{BSTO} / \mathrm{BaM}$ multilayer on alumina. The peaks corresponding to $\mathrm{Al}_{2} \mathrm{O}_{3}, \mathrm{BSTO}$, and $\mathrm{BaM}$ have been indexed and the analysis indicates presence of both BSTO and BaM phases. No evidence of impurity phases were found from XRD measurements. However, some interdiffusion at the interfaces of the multilayer structure cannot be ruled out and XRD will not be able to detect impurities that are present less than $5 \%$ by volume. In Fig. 2 we have presented the

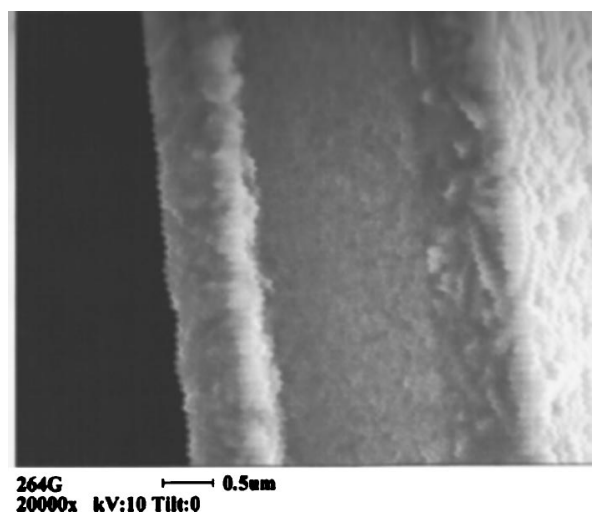

FIG. 2. Cross-sectional SEM image of the BSTO/BAM multilayer showing distinctly visible individual layers.

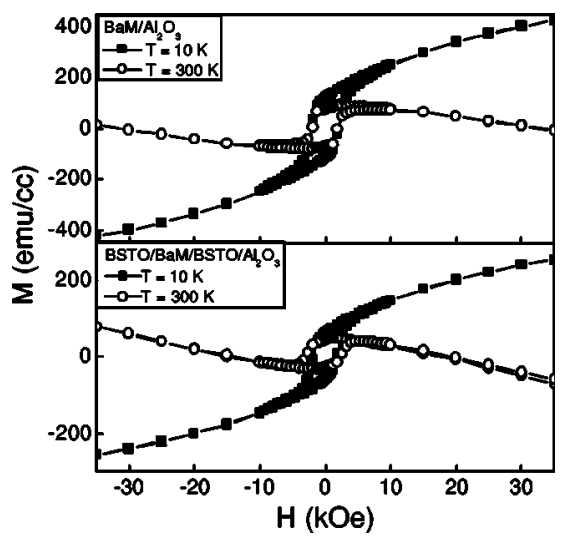

FIG. 3. $M-H$ loops for (i) pure BaM film; (ii) for the postannealed BSTO/ $\mathrm{BaM}$ multilayer grown on a room temperature $\mathrm{Al}_{2} \mathrm{O}_{3}$ substrate at $10 \mathrm{~K}$ and $300 \mathrm{~K}$. A diamagnetic background due to the substrate has been subtracted from the $M-H$ loop data at $300 \mathrm{~K}$.

cross-sectional scanning electron microscope (SEM) image for one of the multilayer samples (with overall thickness of 3 microns). A multilayer structure with well-defined and distinctly visible layers from a coarse grain structure point of view is evident even after annealing. SEM and AFM images (not presented here) show the elongated platelet grains typical of $\mathrm{BaM}$ and the average grain size of the $\mathrm{BaM}$ particles is roughly of the order of $1.2 \mu \mathrm{m} \times 0.3 \mu \mathrm{m}$. The shape and size of the grains depends on the preparation conditions which determines the magnetic properties of the films. It is reported that the addition of BSTO promotes the grain size and decreases the coercivity. ${ }^{15}$ The grain size of the BaM particles for these multilayer films is very large for recording applications as one needs to have smaller grains on the order of $100 \AA$ for maximum recording density. ${ }^{11}$ But for the microwave properties the dielectric constant and the permeability seem to increase with increase in the grain size. ${ }^{15}$

Magnetization measurements were performed at various temperatures using a PPMS equipped with a $7 \mathrm{~T}$ superconducting magnet. The top panel of Fig. 3 shows the magnetization measurements of the pure BaM films grown in the same conditions. The saturation magnetization $\left(M_{S}\right)$ calculated from the moment (emu) taking into account the volume of the films is about $420 \mathrm{emu} / \mathrm{cm}^{3}$ and matches well with reported values. ${ }^{12,13}$ The accuracy of the value is influenced by the error in estimating the thickness of the films. The coercivity $\left(H_{C}\right)$ is observed to be around $2000 \mathrm{Oe}$. In the bottom panel of Fig. 3 we have shown the in-plane $M-H$ loops measured at $10 \mathrm{~K}$ and $300 \mathrm{~K}$ for a BaM/BSTO multilayer. The presence of large coercivity $\left(H_{C} \sim 1800 \mathrm{Oe}\right)$ at $10 \mathrm{~K}$ is consistent with the hard magnetic nature of the $\mathrm{BaM}$ phase. $\mathrm{BSTO}$ layers and the $\mathrm{Al}_{2} \mathrm{O}_{3}$ substrate contribute to a diamagnetic background, which is reflected in the negative slopes of the $M-H$ curves at higher fields in the room temperature data. This diamagnetic background has been subtracted in the data shown. However, at $10 \mathrm{~K}$ there is a paramagnetic response at high fields which is not due to the substrate material. This type of behavior in the $M-H$ loops of pure $\mathrm{BaM}$ films has been observed and ascribed to the uniaxial anisotropy in the hexaferrite system. ${ }^{13,17}$ The mag- 
netization hysteresis loops are almost the same for magnetic fields applied parallel and perpendicular to the substrate at $10 \mathrm{~K}$.

The saturation magnetization of the multilayer decreases in comparison to the pure BaM films due to the presence of BSTO. The important observation made from these magnetization measurements also is the decrease in coercivity of these multilayers. The coercivity is known to be correlated with the grain size, and it has been reported that coercivity decreases with an increase in grain size. ${ }^{11}$ A decrease in $H_{C}$ with increase in BSTO concentration has been reported in bulk composite samples of Z-type hexaferrite $\left(\mathrm{Ba}_{3}\left(\mathrm{Co}_{0.4} \mathrm{Zn}_{0.6}\right)_{2} \mathrm{Fe}_{23.4} \mathrm{O}_{41}\right)$ (Ref. 15) and is attributed to the decrease in effective magnetocrystalline anisotropy. It is possible that the decrease in saturation magnetization and coercivity in our multilayer films are a result of a similar influence of BSTO layers on the grain growth in neighboring BaM layers. It has also been reported that the increase in BSTO content in the Z-type hexaferrite $\left(\mathrm{Ba}_{3}\left(\mathrm{Co}_{0.4} \mathrm{Zn}_{0.6}\right)_{2} \mathrm{Fe}_{23.4} \mathrm{O}_{41}\right)$ resulted in improved complex permeability and permittivity. ${ }^{15}$ However, it is to be noted that in their case, the amount of BSTO doped is only $1.5 \%$. With up to $50 \%$ BSTO/BaM mixture in our composite multilayer films, we expect a broader range of tunability in the complex impedance spectra which would be very useful for $\mathrm{rf}$ applications. We are in the process of depositing these films in a capacitor structure with $\mathrm{Au}$ electrodes so as to measure the dielectric properties and also the electrical tunability. Our first attempt with a $\mathrm{Pt}$ underlayer and an $\mathrm{Au}$ counter electrode on top of the multilayer, failed because of Pt diffusion across the interface during the postannealing stage. Further research is needed to optimize good metallic underlayers and avoid interdiffusion.

\section{CONCLUSIONS}

Multilayers of BSTO/BaM have been successfully grown for the first time and characterized structurally and magnetically. Hysteresis loops revealed the influence of magnetocrystalline and shape anisotropies associated with the $\mathrm{BaM}$ grain structure. Reductions in the saturation magnetiza- tion and coercivity are observed in the multilayer samples when compared to pure BaM films and ascribed to the possibility of the grain growth in the BaM phase being affected by the presence of BSTO.

\section{ACKNOWLEDGMENTS}

Work at USF is supported by a grant from DARPA through ARO Grant No. DAAD19-03-1-0277. R.H. is a NSF IGERT fellow supported from Grant No. DGE-0221681. Work at Oak Ridge National Laboratory was sponsored by the U.S. Department of Energy, Division of Materials Science. The authors thank Dr. Pankaj Poddar for helpful discussions.

${ }^{1}$ T. Kimura, T. Goto, H. Shintanl, K. Ishlzaka, T. Arima, and Y. Tokura, Nature (London) 426, 55 (2003).

${ }^{2}$ N. Hur, S. Park, P. A. Sharma, J. S. Ahn, S. Guha, and S. W. Cheong, Nature (London) 429, 392 (2004).

${ }^{3}$ W. J. Kim, W. Chang, S. B. Qadri, H. D. Wu, J. M. Pond, S. W. Kirchoefer, H. S. Newman, D. B. Chrisey, and J. S. Horwitz, Appl. Phys. A: Mater. Sci. Process. 71, 7 (2000).

${ }^{4}$ P. Lubitz and F. J. Rachford, J. Appl. Phys. 91, 7613 (2002); C. E. Patton, IEEE Trans. Magn. 24, 2024 (1988).

${ }^{5}$ T. Nakamura and K. Hatakeyama, IEEE Trans. Magn. 36, 3415 (2000).

${ }^{6}$ I. Vendik, O. Vendik, V. Pleskachev, and M. Nikol'ski, IEEE Trans. Appl. Supercond. 13, 716 (2003).

${ }^{7}$ P. Padmini, T. R. Taylor, M. J. Lefevre, A. S. Nagra, R. A. York, and J. S. Speck, Appl. Phys. Lett. 75, 3186 (1999).

${ }^{8}$ W. Chang, J. S. Horwitz, A. C. Carter, J. M. Pond, S. W. Kirchefer, C. M. Gilmore, and D. B. Chrisey, Appl. Phys. Lett. 74, 1033 (1999).

${ }^{9}$ J. Im, O. Auciello, P. K. Baumann, S. K. Streiffer, D. Y. Kaufman, and A. R. Kraus, Appl. Phys. Lett. 76, 625 (2000).

${ }^{10}$ M. Matsuoka, Y. Hoshi, M. Naoe, and S. Yamanaka, IEEE Trans. Magn. MAG-18, 1119 (1982).

${ }^{11}$ T. L. Hylton, M. A. Parker, M. Ullah, K. R. Coffey, R. Umphress, and J. K. Howard, J. Appl. Phys. 75, 5960 (1994).

${ }^{12}$ S. R. Shinde, R. Ramesh, S. E. Lofland, S. M. Bhagat, S. B. Ogale, R. P. Sharma, and T. Venkateshan, Appl. Phys. Lett. 72, 3443 (1998).

${ }^{13}$ S. Capraro, J. P. Chatelon, H. Joisten, M. Le Berre, B. Bayard, D. Barbier, and J. J. Rousseau, J. Appl. Phys. 93, 9898 (2003).

${ }^{14}$ R. Hajndl, J. Sanders, H. Srikanth, and N. J. Dudney, J. Appl. Phys. 93, 7999 (2003).

${ }^{15}$ P. Huang, L. J. Deng, J. X. Xie, D. F. Liang, and L. Chen, J. Magn. Magn. Mater. 271, 97 (2004).

${ }^{16}$ M. S. Pinho, M. L. Gregori, A. S. Carvalho, R. C. R. Nunes, and B. G. Soares, Microwave Opt. Technol. Lett. 32, 326 (2002).

${ }^{17}$ Xiaoyu Sui and Mark H. Kryder, Appl. Phys. Lett. 63, 1582 (1993). 